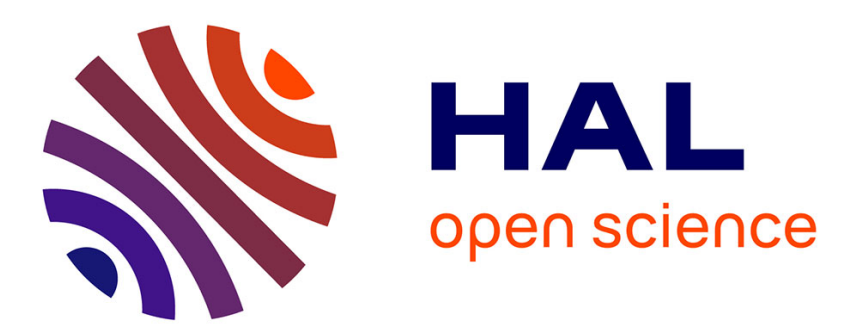

\title{
Influence of the quasiparticle spectral weight in FeSe on spectroscopic, magnetic, and thermodynamic properties
}

H. Cercellier, Pierre Rodière, Pierre Toulemonde, C. Marcenat, T. Klein

\section{To cite this version:}

H. Cercellier, Pierre Rodière, Pierre Toulemonde, C. Marcenat, T. Klein. Influence of the quasiparticle spectral weight in FeSe on spectroscopic, magnetic, and thermodynamic properties. Physical Review B, 2019, 100 (10), pp.104516. 10.1103/PhysRevB.100.104516 . hal-02320721

\section{HAL Id: hal-02320721 \\ https://hal.science/hal-02320721}

Submitted on 16 Nov 2020

HAL is a multi-disciplinary open access archive for the deposit and dissemination of scientific research documents, whether they are published or not. The documents may come from teaching and research institutions in France or abroad, or from public or private research centers.
L'archive ouverte pluridisciplinaire HAL, est destinée au dépôt et à la diffusion de documents scientifiques de niveau recherche, publiés ou non, émanant des établissements d'enseignement et de recherche français ou étrangers, des laboratoires publics ou privés. 


\title{
Influence of the quasiparticle spectral weight in FeSe on spectroscopic, magnetic, and thermodynamic properties
}

\author{
H. Cercellier, ${ }^{1, *}$ P. Rodière,${ }^{1}$ P. Toulemonde,${ }^{1}$ C. Marcenat,,${ }^{1,2}$ and T. Klein ${ }^{1}$ \\ ${ }^{1}$ Université Grenoble Alpes, CNRS, Grenoble INP, Institut Néel, F-38000 Grenoble, France \\ ${ }^{2}$ CEA, INAC, PhELIQS, LATEQS, F-38000 Grenoble, France
}

(Received 22 February 2019; revised manuscript received 23 August 2019; published 16 September 2019)

\begin{abstract}
We report on an extensive experimental and numerical study of the low-energy electronic properties of superconducting FeSe single crystals, using point-contact Andreev reflection Spectroscopy (PCAR), specific heat, and London penetration depth measurements. Taking explicitly into account Fermi surface anisotropy and recently suggested orbital-selective quasiparticle spectral weights $Z_{i}\left(i=d_{x y}, d_{x^{2}-y^{2}}, d_{x z}, d_{y z}, d_{z^{2}}\right)$, our calculations quantitatively account for all our measurements as well as data from the literature, assuming that $Z_{y z}>Z_{x z}>Z_{x y}$. This study confirms the picture of a highly different quantum coherence of the Fe orbitals at the Fermi energy. In particular, the normal state properties (Sommerfeld coefficient and zero-temperature London penetration depth) strongly depend on the $Z_{i}$ values, which seem to be significantly sensitive to disorder.
\end{abstract}

DOI: 10.1103/PhysRevB.100.104516

\section{INTRODUCTION}

FeSe is a particularly interesting iron-based superconductor, being simply a $c$-axis stacking of FeSe layers without any charge reservoirs. Moreover, even if it undergoes a structural phase transition similar to that found in all other iron-based compounds [1], this system does not order magnetically. It has been suggested that superconductivity competes with an exotic charge ordered state with orbital nematicity lowering rotational symmetry without breaking the translational one [2-4]. Though still debated, many theoretical investigations of FeSe in its normal state point to an interplay of the Hund coupling and orbital-dependent strong Coulomb interactions as the driving force for nematic ordering, resulting in very small and highly orbitally ordered Fermi pockets in the nematic state [5,6]. Recent angle-resolved photoemission spectroscopy (ARPES) studies have evidenced Hubbard bands and strongly renormalized quasiparticle (QP) dispersions at the Fermi energy, with a strongly anisotropic spectral weight in the normal state $[7,8]$. Following this idea, Kreisel et al $[9,10]$ introduced an orbital-selective QP spectral weight (SW) $\mathbf{Z}(\mathbf{k})$ in the superconducting pairing. This pairing, mediated by dressed spin fluctuations, results in a strongly anisotropic nodeless $s_{ \pm}$state, in excellent agreement with low-temperature Bogoliubov quasiparticle inferterence (BQPI) measurements [11].

Even if the presence of nodes as well as the $s_{ \pm}$symmetry remain debated, many thermodynamic, transport and magnetic studies have confirmed the anisotropic multigap character of FeSe [12-21]. However, a major weakness of "standard" multigap fits to the data is the weight of each gap in the total fits. In most of the literature (well beyond the case of $\mathrm{FeSe}$ ), the contribution of each gap is a free parameter, and very few attempts have been made to link the fitted weights to

*herve.cercellier@neel.cnrs.fr the actual band structure of the compounds. In this paper we show that, beyond the use of realistic band structure calculations, and gap functions from the literature, one can calculate quantitatively various properties of FeSe with the introduction of appropriate quasiparticle spectral weights (QPSWs). This includes normal state properties (i.e., Sommerfeld coefficient and zero-temperature London penetration depth) as well as three fundamental superconducting properties of Fese: the temperatures dependences of both the superfluid density and the specific heat, and also the point-contact Andreev reflection (PCAR) spectra.

The weight of each band (gap) is then not a free parameter anymore and we show that QPSWs $<1$ are necessary to obtain a consistent description of those fundamental properties, with $Z_{x y}<Z_{x z} \ll Z_{y z}<1$, hence supporting orbital selectivity of correlations and quasiparticle coherence as a key feature of FeSe. Our calculations quantitatively account for all our measurements as well as data from the literature, by slightly adjusting the QPSW values. Finally, the influence of disorder on the QPSW is also discussed.

\section{EXPERIMENTS}

High quality FeSe single crystals were grown by chemical vapor transport [22]. The typical structural and transport properties of the samples can be found in Ref. [22]. The high quality of the crystals was attested by the well defined specific heat anomaly at $T_{c}$ (see Ref. [22] and Fig. 3) as well as the sharp drop of the magnetic penetration depth [see right inset in Fig. 2(a)] and the previously observed quantum oscillations [23]. In this study, the measured critical temperatures were in the range $T_{c} \sim 8.5 \pm 0.4 \mathrm{~K}$, depending on sample and experimental technique, as expected from the measured residual resistivity ratio $\mathrm{RRR} \sim 15-20$ [24]. London penetration depth measurements were performed using the tunnel-diode-oscillator (TDO) technique [25]. Specific heat measurements were performed down to $0.5 \mathrm{~K}$ and in 


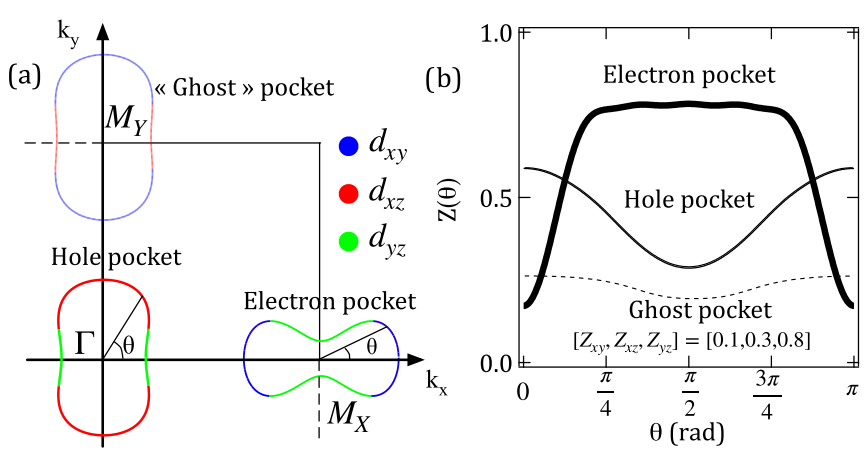

FIG. 1. (a) Schematic Fermi surface of FeSe used in the model calculations (not to scale). The "ghost pocket" around the $M_{Y}$ point carries a small spectral weight and is supposed to carry a zero superconducting gap. (b) Anisotropy of the quasiparticle spectral weight on the three pockets for the indicated ORSW $\left(Z_{i}\right)$ values (see Fig. 5 and Sec. IV). The same twofold anisotropy is taken for the SW and the gaps.

magnetic fields up to $18 \mathrm{~T}$, using an AC heating technique. The superconducting contribution to the electronic specific heat is given by $\Delta C / T=\left[C_{\text {tot }}(T, H=0)-C_{\text {tot }}(T, H=18 T)\right] / T$.

PCAR measurements were carried out on freshly cleaved surfaces by the needle-anvil technique [26-28]. Constrictions were made between an Au tip and the $a b$ plane of the FeSe single crystals, with current injection mainly along the $c$ axis. Contact resistances were in the range 1-20 $\Omega$. The differential conductance was measured between 1.6 and $10 \mathrm{~K}$ by the standard lock-in technique.

\section{CALCULATIONS}

The basic parameters of the model calculations are shown in Fig. 1. Use is made of the tight-binding (TB) low-energy band structure $\left(E_{k}\right)$ introduced by Kreisel and co-workers $[9,11]$. The wave functions are defined on a five- $3 d$-orbital basis: $\Psi(\mathbf{k})=\sum_{i} \mathbf{a}_{\mathbf{i}}(\mathbf{k}) \phi_{i}$, where $i=d_{x y}, d_{x^{2}-y^{2}}, d_{x z}, d_{y z}, d_{z^{2}}$. An approximate spectral function $A(\mathbf{k}, \omega) \approx \mathbf{Z}(\mathbf{k}) \delta\left(\omega-E_{k}\right)$ is introduced, where $\mathbf{Z}(\mathbf{k})$ is the quasiparticle spectral weight (QPSW) and $\delta\left(\omega-E_{k}\right)$ the Dirac function. The weight $\mathbf{Z}(\mathbf{k})<1$ defines the contribution of each Fermi wave vector to the physical properties. Parts of the Fermi surface with small $\mathbf{Z}(\mathbf{k})$, such as the $\delta$ electron pocket ["ghost pocket" in Fig. 1(a)], of dominant incoherent $d_{x y}$ character (it is barely visible in ARPES), are supposed to carry a zero superconducting gap (this pocket will still contribute to the normal state properties). In contrast $\mathbf{Z}(\mathbf{k})$ is maximum for the $d_{y z}$ orbitals (green parts of the electron pocket around $\theta= \pm \pi / 2$ and the hole pocket around $\theta=0[\pi]$; see Fig. 1 ).

As the TB model depends weakly on the wave vector along the $c$ axis, the QPSW anisotropies are chosen to vary only in the $a b$ plane, and are parametrized by the angle $\theta$ with respect to the $k_{x}$ axis [see Fig. 1(a)]. We assume that the QPSW can also be decomposed on the $3 d$-orbital basis: $\mathbf{Z}(\mathbf{k}) \rightarrow \mathbf{Z}(\theta)=\sum_{i} Z_{i}\left|\mathbf{a}_{\mathbf{i}}(\theta)\right|^{\mathbf{2}}$, where the $Z_{i}$ are the orbitalresolved spectral weights (ORSWs), being the free parameters of the model. Within the energy range of interest, taking the ORSW as constants is a good approximation [29]. At

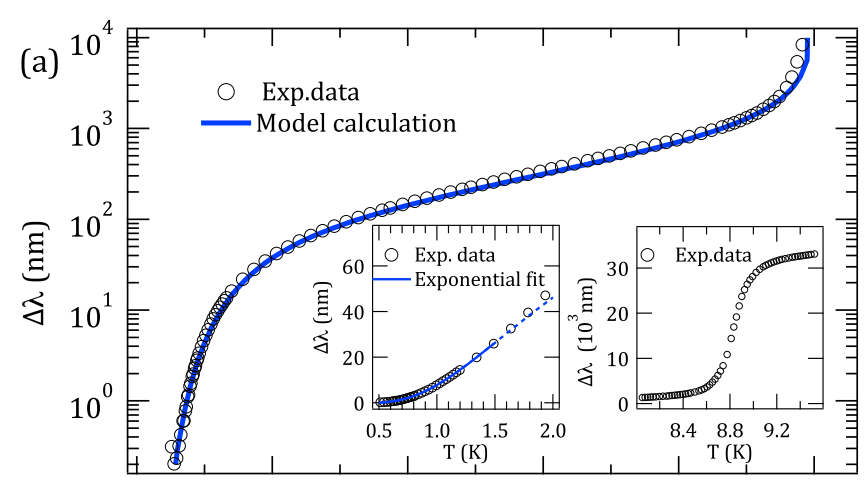

(b)
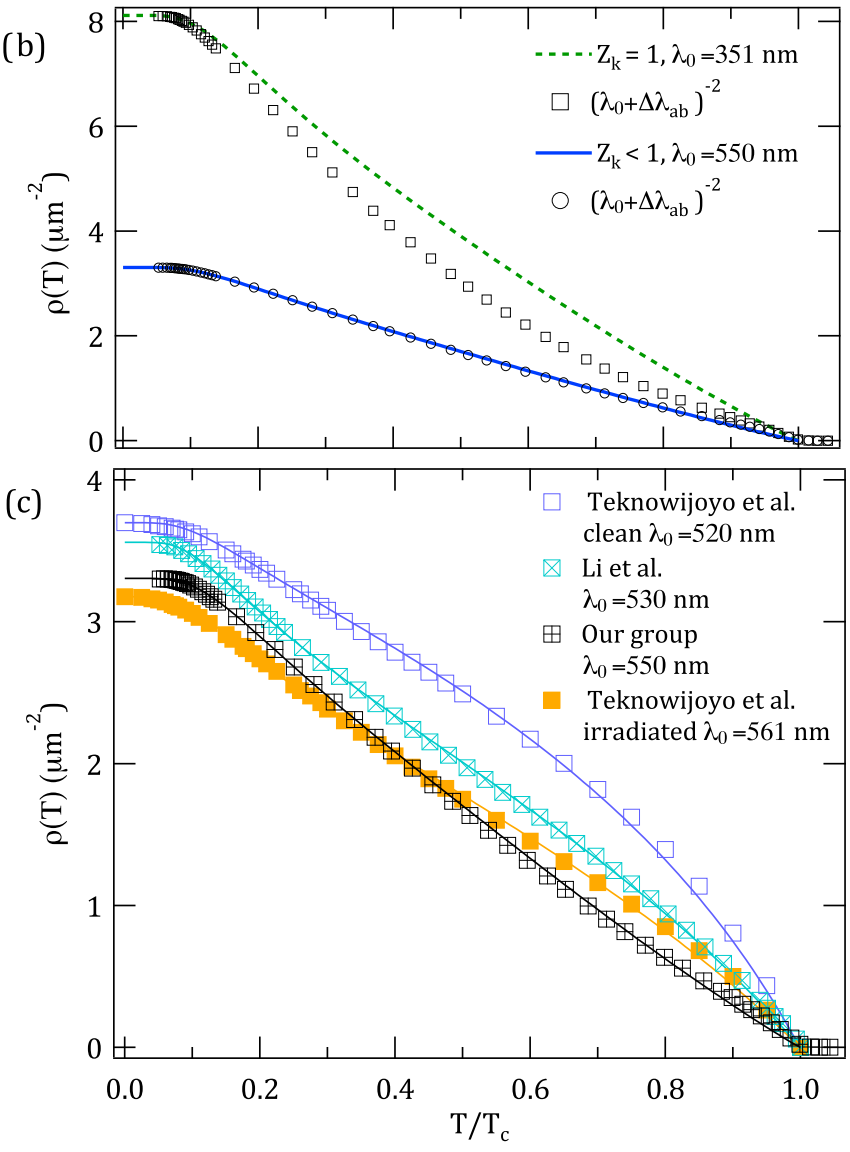

FIG. 2. (a) Temperature dependence of the London penetration depth (open symbols) and fit to the data (full line; see text for details). The low- $T$ behavior clearly displays an exponential dependence, indicating the presence of a fully open (small) gap. (b) Superfluid density, calculated (thick lines) and reconstructed from $\Delta \lambda$ measurements (symbols; see text for details). (c) Comparison of several data sets from the literature $[19,20]$ (symbols) and their respective model calculations (full lines). The ORSW and gap values are reported in Figs. 5 and 6, respectively.

the Fermi energy the $d_{x^{2}-y^{2}}$ and $d_{z^{2}}$ orbitals have a small contribution to the total spectral weight. We will then consider only the relevant $d_{x y}, d_{x z}$, and $d_{y z}$ orbitals. Figure 1(b) displays the anisotropy of the QPSW for a significant set of ORSWs $\left[Z_{x y}, Z_{x z}, Z_{y z}\right]=[0.1,0.3,0.8]$, obtained from fits to the data of Refs. [9-11,19,20] and our PCAR measurements (see below). In this case the dominant $d_{y z}$ and the weak $d_{x y}$ SWs lead to the same twofold anisotropy for the SW and the gaps [15] 
(see Fig. S5 in the Supplemental Material [30] for an illustration of this angular dependence), and only the gap extrema were adjusted to fit the data. The temperature dependence of the gaps was chosen as $\Delta(T)=\Delta_{0} \tanh \left(\alpha \sqrt{T_{c} / T-1}\right)$, with $\alpha=1.7 \pm 0.15$ depending on samples (see Fig. S5 in the Supplemental Material [30]) for both London penetration depth and specific heat measurements.

The normal state Sommerfeld coefficient and zero temperature London penetration depth then strongly depend on the band structure and ORSW values, which are hence strongly constrained by those normal state properties (see Supplemental Material for details about the numerical calculations [30]). The temperature dependence of the specific heat and superfluid density can then be calculated without any arbitrary adjustment of the relative contribution of each band.

\section{RESULTS AND DISCUSSION}

\section{A. Superfluid density}

The relative variation of the London penetration depth (in the $a b$ plane), $\Delta \lambda$, and the corresponding calculation [30] are shown in Fig. 2(a). At low temperature $[T<2 K$; see inset of Fig. 2(a)], the data can be well fitted by $\Delta \lambda \propto$ $\Delta_{S} / T \exp \left(-\Delta_{S} / T\right)$, indicating the presence of a nodeless gap of amplitude $\Delta_{S} \simeq 0.3 \mathrm{meV} \simeq 0.4 k_{B} T_{c}$, in agreement with the results of Ref. [20] obtained by the same technique. The data can be very well fitted on the whole temperature range by using gaps and $Z_{i}$ reasonably close to the value predicted theoretically [9] and measured by scanning tunneling microscopy (STM) [11] (see Fig. 5 and the discussion below). The contribution from the electron pocket to the total superfluid density is then of the order of $80 \%$ of the signal, as this pocket carries both the largest Fermi velocities (see Supplemental Material, Fig. S1) and the largest SW ( $d_{y z}$ orbitals).

The experimental superfluid density was then reconstructed from the measured shift $\Delta \lambda$ and the calculated $0 \mathrm{~K}$ penetration depth $\left[\lambda_{0} \sim 550 \mathrm{~nm}\right.$; see Eq. (2) in Supplemental Material [30] and open circles in Fig. 2(b)] as $\rho(T)=\left(\lambda_{0}+\right.$ $\left.\Delta \lambda_{a b}\right)^{-2}$. As a comparison, the superfluid density calculated with the same gap structure, but with $\mathbf{Z}(\theta)=1$ (corresponding to the smallest possible $\lambda_{0} \sim 350 \mathrm{~nm}$ value within the model) is also given in Fig. 2(b) (open squares). A clear discrepancy with the theoretical curve is observed in this latter case, mainly arising from the too small $\lambda_{0}$ value.

Figure 2(c) displays the temperature dependence of the superfluid density from various studies in the literature, in comparison with the one obtained in the present work. As shown, both $\lambda_{0}$ and the overall shape of the $T$ dependence vary from one sample to another, but all data can be well reproduced using the procedure described above, by introducing slightly different $Z_{i}$ values. A first case considered is the penetration depth measurements by Li et al. [19]. With a measured low-temperature QP mean free path $l=55 \mu \mathrm{m}$, the studied system was clearly in the clean limit. Compared to our data, a good fit is obtained with similar $Z_{x y}$ and $Z_{x z}$, but a slightly larger $Z_{y z}$ (leading to $\lambda_{0} \sim 530 \mathrm{~nm}$, close to the value for our sample). One can then reasonably assume that our samples are also in the clean limit (PCAR measurements confirm this assumption; see below).
A second case is the effect of disorder studied by Teknowijoyo et al. in Ref. [20]. We find a reasonable $\lambda_{0} \sim 520 \mathrm{~nm}$ value for the clean sample, with a very low $Z_{x y}$ and a high $Z_{y z}$. In the irradiated sample [20], there is a strong decrease of $Z_{y z}$ from 0.8 to 0.5 and an increase of $Z_{x y}$ from 0.1 to 0.3 (see Fig. 5 below). This results in $Z_{i}$ (and gaps) very similar to those measured in our sample and by Li et al. [19]. Those measurements hence highlight the influence of sample quality on the SW values (see discussion below). Irradiation was shown to weaken nematic ordering [20]. Besides, systematic studies of doping in FeSe have shown that the nematic splitting of $d_{x z}$ and $d_{y z}$ orbitals is suppressed either by isovalent substitution of Se by $\mathrm{S}$ or $\mathrm{K}$ dosing [31]. As a result one expects the orbital spectral weights to become more similar in disordered samples, as there is no reason to promote one type of $d$ orbital over the others in the absence of nematicity, in agreement with the data.

The fitted gap minima $\Delta_{i}^{0} \lesssim 0.5 k_{B} T_{c}$ are only weakly dependent on sample quality. On the other hand, the obtained gap maxima are much larger in Teknowijoyo's clean sample [20] (see Fig. 6 below), where $Z_{y z}$ is large and $Z_{x y}$ is small. The qualitative dependence of the gap functions on $d_{y z}$ and $d_{x y} \mathrm{SW}$ is in agreement with the model of orbitally dependent pairing [9].

\section{B. Specific heat}

The absolute superconducting contribution to the specific heat $\Delta C / T=\left(C_{S}-C_{N}\right) / T$, measured on our samples and taken from various studies of the literature [18,21], are displayed in Fig. 3. The measured Sommerfeld coefficient is $\gamma \sim$ $5.5 \mathrm{~mJ} / \mathrm{mol} \mathrm{K}^{2}$ for our sample, and up to $\gamma \sim 6.9 \mathrm{~mJ} / \mathrm{mol} \mathrm{K}^{2}$ in sample 1 of Hardy et al. with the highest reported $T_{c} \sim$ 9.1 K [21]. Reasonable agreement is found with other previous measurements [12,15,22]. The specific heat jump at $T_{c}$, $\Delta C / T_{c}$, decreases with decreasing $T_{c}$ but remains higher than the BCS value $\Delta C_{\mathrm{BCS}} / T_{c}=1.43$, revealing the existence of a superconducting gap larger than the BCS single gap value $\Delta \gtrsim 1.76 k_{B} T_{c}$. As shown, the calculations are in excellent agreement with the measured data introducing the orbitally resolved spectral weight values displayed in Fig. 5. Note that, as already mentioned above, the orbital SWs are strongly constrained by the normal state properties (here the Sommerfeld coefficient; see Eq. (1) in the Supplemental Material [30]) and the effect of QPSW on $\Delta C / T$ is again dramatic: with $\mathbf{Z}(\theta)=1$ it would lead to a Sommerfeld coefficient $\gamma=$ $10.5 \mathrm{~mJ} / \mathrm{mol} \mathrm{K}^{2}$, which is much larger than any published value. In this case the calculated curve (green dashed line in Fig. 3) completely overestimates the experimental specific heat.

Quite different temperature dependences of the specific heat can be observed below $\sim T_{c} / 3$. Much of the controversy about the low-energy gap structure of FeSe is then related to the observation of a linear behavior of the low-temperature specific heat [21] and/or the presence (or absence) of a hump around $1 \mathrm{~K}[14,17]$, making it difficult to determine the "true" superconducting gap of "ideal" FeSe (several specific heat studies from the literature are compared in [21], clearly showing that there is no clear relationship between a higher $T_{c}$ and the existence/nonexistence of the $1 \mathrm{~K}$ humplike behavior). We show here that the various low-temperature behaviors can be 


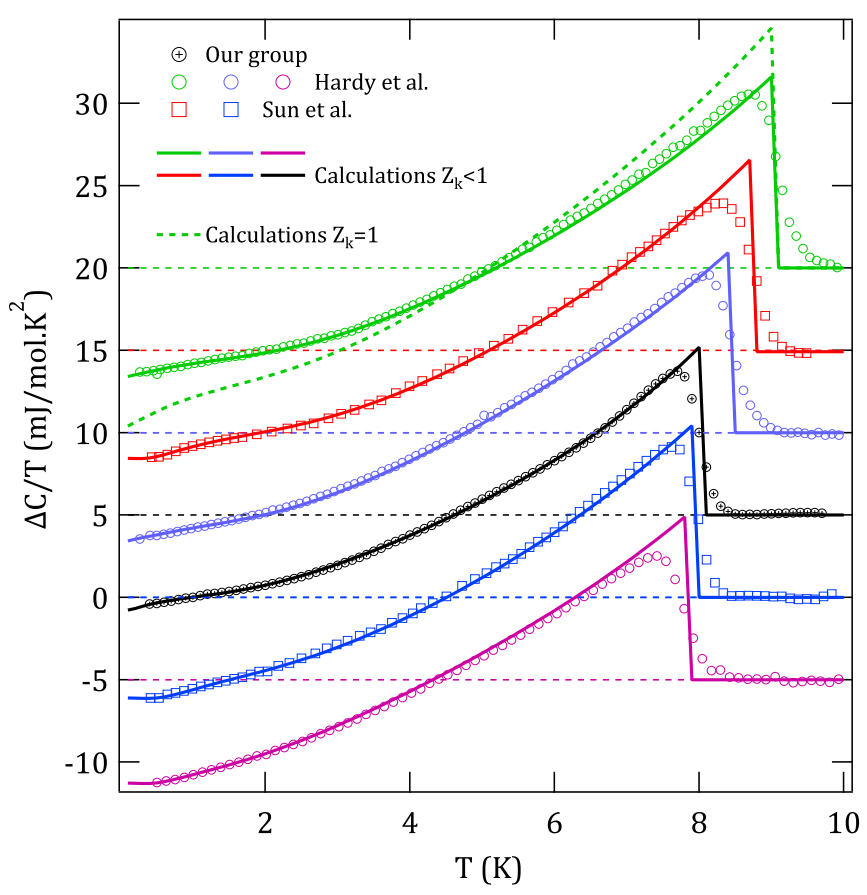

FIG. 3. Specific heat measured on various samples with different critical temperatures (decreasing $T_{c}$ from top curve to bottom curve). The curves are systematically shifted by $5 \mathrm{~mJ} / \mathrm{mol} \mathrm{K}^{2}$ for clarity. Curves labeled as "Hardy et al." are taken from Ref. [21] and the curves labeled as "Sun et al." are taken from Ref. [18]. Full lines are the model calculations, taking into account QP weights displayed in Fig. 5 (see text for details). The dashed line is the model calculation with unity QP weights. In this case the absolute value of the specific heat is clearly overestimated. The values of the gap extrema deduced from the model calculations are displayed in Fig. 6(a).

accounted for without drastic changes in the gap functions, simply by changing the ratio of $d_{x y}$ to $d_{x z}$ spectral weights. Indeed, nearly linear behavior [21] can be obtained with a high SW ratio $Z_{x y} / Z_{x z} \approx 0.6$, supporting the prediction by Kreisel et al [9] and Sprau et al [11] that the superconducting (SC) gaps have nodes if the spectral weight distribution is neglected, and become nodeless otherwise. Similarly a hump around $1 \mathrm{~K}[14,17,18]$ is clearly visible for $Z_{x y} / Z_{x z} \approx 0.3$ (red curve). However, such sizable differences in the $d_{x y / x z} \mathrm{SW}$ ratio are difficult to explain as an intrinsic effect, and the possibility that this hump could be an extrinsic effect due to a small amount of magnetic impurities cannot be excluded [21]. Still, the specific heat measurements hence clearly confirm the hierarchy previously inferred from London penetration measurements: $Z_{y z} \gg Z_{x z}>Z_{x y}$, in excellent agreement with the theoretical predictions [9] (see below for a discussion on the evolution of the gaps with $T_{c}$ ).

\section{Point-contact Andreev reflection}

The normalized differential conductivities $\left(G=R_{N}^{-1}\right.$ $d I / d V)$ of two typical Au/FeSe $c$-axis junctions at $T=1.6 \mathrm{~K}$ are displayed in Fig. 4. Note that even though the current is mainly injected along the $c$ axis of the crystal, the $a b$-plane anisotropy of the SC gaps and the SW still can strongly influence the measured differential conductance due to wave-

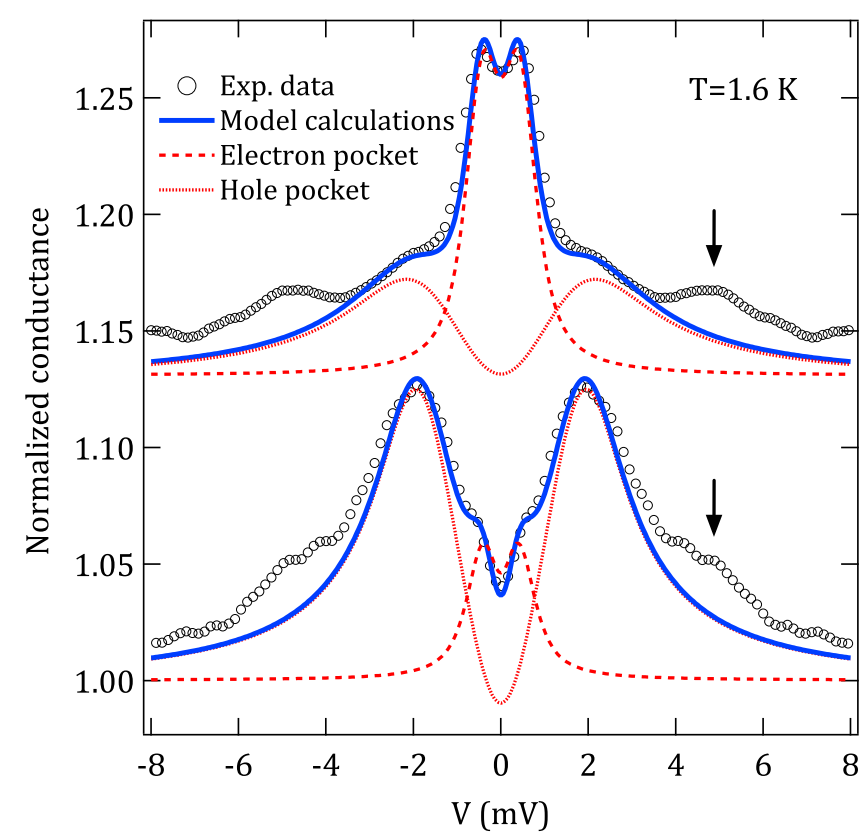

FIG. 4. Two examples of $c$-axis PCAR spectra measured at $1.6 \mathrm{~K}$. The contributions from the hole and the electron pockets are drawn as dotted and dashed lines, respectively. The hump indicated by the arrow is most probably a strong coupling signature of the previously observed spin resonance (see text). Fitting parameters: upper curve $\Lambda_{e}=0.15, \Lambda_{h}=0.01, \Gamma_{e}=0.12 \mathrm{meV}, \Gamma_{h}=1.25 \mathrm{meV}$; lower curve $\Lambda_{e}=0.1, \Lambda_{h}=0.27, \Gamma_{e}=0.05 \mathrm{meV}, \Gamma_{h}=0.37 \mathrm{meV}$.

vector conservation in the $a b$ plane across the junction. More precisely, in our case the measured differential conductance results from an integration of all low-energy excitations along the projection of the Fermi surface on the $a b$ plane (see Supplemental Material [30] for more details or Refs. [27,28] for a complete review of PCAR on multigap systems). As a result, for highly anisotropic Fermi surfaces and/or SC gaps, the $c$-axis differential conductance will exhibit structures reminiscent of the characteristic energy scales in the $a b$ plane.

Here, both curves exhibit a low bias anomaly at $V \approx$ $0.4 \mathrm{meV}$ and a broad shoulder at $V \approx 2 \mathrm{meV}$. This double structure is reminiscent of multigap systems [27,28] but, even though a large gap $\Delta_{L} \approx 2 \mathrm{meV}$ has been evidenced in several STM studies [14,32-34], to our knowledge this is the first observation of low-bias peaks. As will be discussed more extensively later, the observation of such well-resolved structures at $T=1.6 \mathrm{~K}$ suggests that the lifetime broadening of low energy quasiparticles is very small, in agreement with [19]. The data have been fitted to the orbital-dependent SW model using the three-dimensional Blonder-TinkhamKlapwijk (BTK) model [27,28,35]. The calculations take into account the full three-dimensional Fermi surface of FeSe, and a spherical Fermi surface is taken for the Au electrode. The calculation also involves two transmission barrier heights $\Lambda_{e}$ and $\Lambda_{h}$ for the electron and hole pockets, respectively, and the corresponding lifetime broadenings $\Gamma_{e}$ and $\Gamma_{h}$. In order for the model to be physically consistent, the spectral functions have also been modified as Lorentzian peaks of width $\Gamma_{e}$ and $\Gamma_{h}$ and spectral weight $\mathbf{Z}(\theta)$ (see Supplemental Material Figs. S1 and S4 [30]). The fits to the data are shown as thick blue lines, 


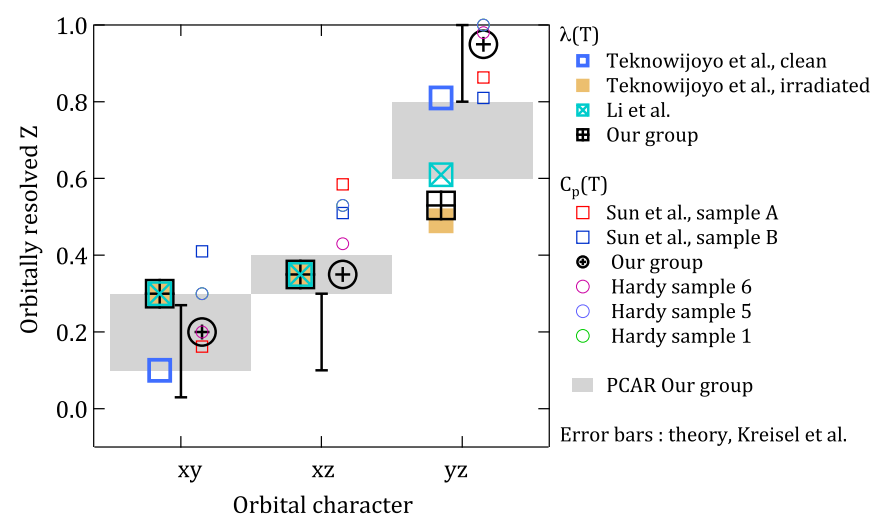

FIG. 5. Orbitally resolved spectral weights obtained from the fits presented in this study. The error bars are taken from Ref. [10] and give a theoretical range of $\mathrm{SW}$ values reproducing the experimental gap functions.

together with the relative contributions from the two Fermi pockets. The electron contribution is peaked around zero bias, due to a subtle and very anisotropic distribution of the Fermi velocities on this pocket [30]. The hole contribution has a much more standard shape, as expected from the lower SW and Fermi velocity anisotropies on this band [Fig. 1(b)].

The geometrical coefficients reflect the quality of the junction, whereas the lifetime broadenings reflect both the quality of the junction and the intrinsic properties of the electrodes. Note that $\Lambda_{e}$ and $\Lambda_{h}$ are not independent: their ratio influences the relative contribution of each Fermi pocket in the total signal. As measurements were systematically carried out on freshly cleaved surfaces, the two coefficients were expected to be small, as verified during the fitting of the data. Some examples of data fits with the corresponding fit parameters are shown in Supplemental Material Fig. S4 [30]. A small geometrical barrier generally corresponds to a clean interface, so that the electron lifetime broadening should reflect mainly the intrinsic properties of the sample. As a matter of fact, all data sets were fitted with $\Gamma_{e} \lesssim 0.1 \mathrm{meV}$, which corresponds to a large electron mean free path $\hbar v_{F} / \Gamma_{e} \gtrsim 1 \mu \mathrm{m}$, consistent with the measurements of Ref. [19] and with the observation of sharp quasiparticle peaks on the electron pocket in ARPES $[7,8]$. Hence the sharp contribution from the electron pocket results from a combination of its very peculiar electronic structure and a high junction transparency. In the tunneling limit $\Lambda_{e}, \Lambda_{h} \gg 1$ (not shown), we have checked that this contribution is exponentially damped and mainly the hole contribution is visible, as observed in STS spectra from the literature $[14,32,33]$.

Some humps are also visible at biases $V_{h} \approx 5 \mathrm{meV}$, which are not accounted for in the calculations (see black arrows in Fig. 4). Such a feature, characteristic of the electronic coupling to a bosonic mode, has been reported in STM measurements on FeSe [36]. A plausible interpretation of these structures is the strong-coupling signature of the spin resonance evidenced by neutron scattering studies [37]. The same type of spin resonance has been observed in many pnictides and chalcogenides, and the possibility of observing it in PCAR spectra has been extensively discussed. According to the Eliashberg strong coupling theory, a bosonic mode with
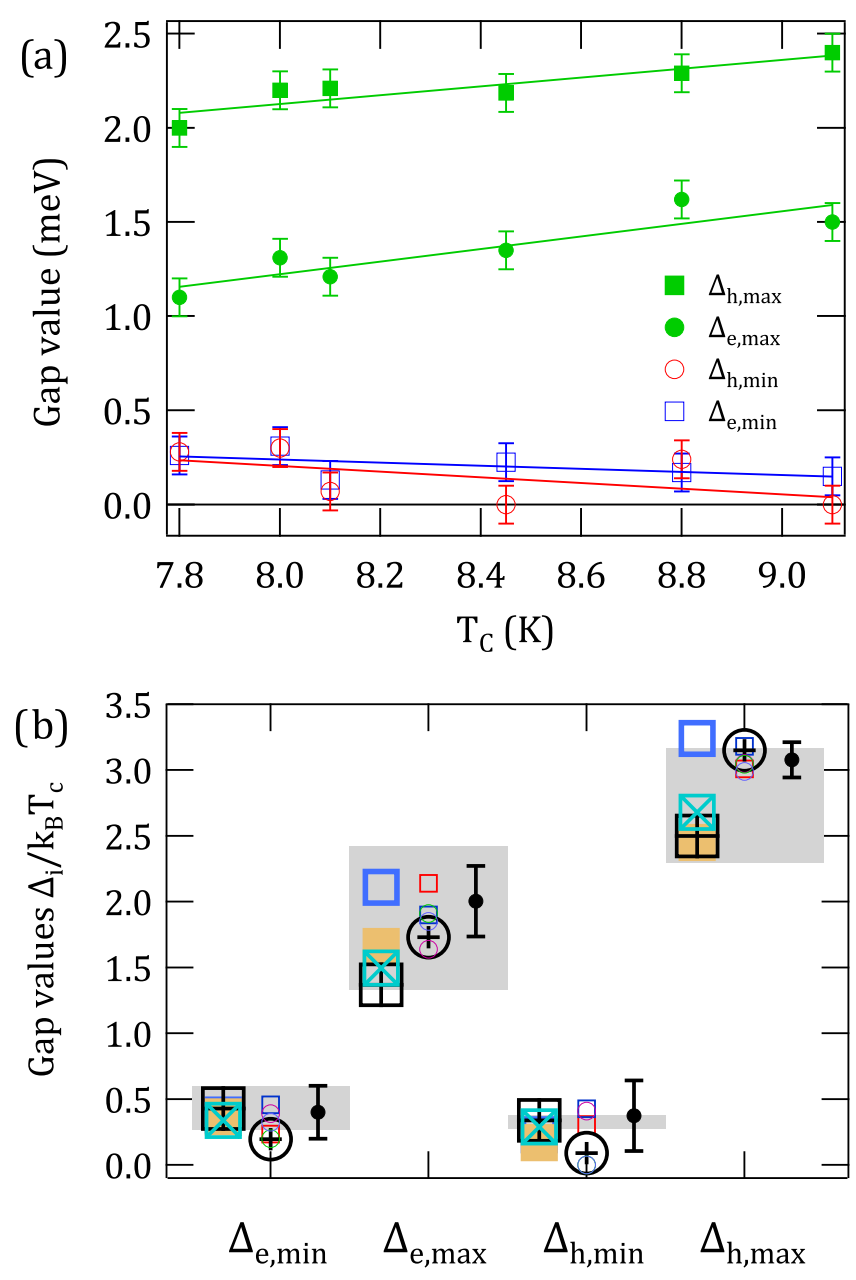

FIG. 6. (a) Gap values deduced from fits to the specific heat data (see Fig. 3) as a function of $T_{c}$. (b) Summary of the gap values deduced from fits to all data (Figs. 2 and 3; see Fig. 5 for the symbol correspondence). For the PCAR measurements (Fig. 4) the grey area represents the error bars, defined as the statistical standard deviation computed on 17 different data sets. The black circles with error bars are the reference measurements from Sprau et al. [11].

characteristic energy $\Omega(T)$ shows up in the conductance at voltage $e V_{\Omega} \approx \Delta(T)+\Omega(T)[27,28]$. In our case, at $1.6 \mathrm{~K}$, $\Omega \sim 4 \mathrm{meV}$, and $\Delta \approx 2 \mathrm{meV}$, leading to $V_{\Omega} \sim 6 \mathrm{mV}$, in good agreement with $V_{h}$. The study of this strong-coupling feature is beyond the scope of this paper.

Many different samples were measured, and all data sets measured at temperatures below $T_{c} / 3$ were fitted as described above. A statistical analysis of the results of these fittings allowed us to give statistical error bars for the spectral weights and SC gaps. They appear as gray shaded areas in Figs. 5 and 6 , respectively. Above $T_{c} / 3 \approx 3 \mathrm{~K}$ the thermal smearing of the spectra prevents a quantitative accurate fitting of the electron pocket gap (i.e., the low-bias signal). On the other hand, the more standard shape of the hole pocket contribution allowed us to fit its temperature dependence with the standard 2D BTK model. The resulting curve for the hole pocket gap maxima follows the same $\Delta(T)$ law used for the penetration depth and specific heat calculations, as can be seen in Supplemental Material Fig. S5 [30]. 


\section{FINAL DISCUSSION AND CONCLUSION}

The orbital spectral weights deduced from our study are summed up in Fig. 5. According to the general idea of orbitally selective SC pairing, the Fermi wave vectors carrying the largest SW must coincide with the gap maxima. All the fits performed in this work lead to $Z_{y z}>Z_{x z}>Z_{x y}$, which is the required condition for a matching of gap and SW maxima. Our results are in remarkable agreement with two recent ARPES studies [38]. Compared to the work of Kreisel et al. [10] (error bars in Fig. 5), our study clearly confirms the dominant weight of the $d_{y z}$ orbitals.

The hole and electron pocket superconducting gaps deduced from the fits to the specific heat data of Fig. 3 are displayed in Fig. 6(a). Even though our calculations can not conclude with certainty about the presence of point- or line nodes in "high $T_{c}$ " samples, fully open gaps are robust features of the lowest $T_{c}$ samples (note that whether the gap is nodal or not will not change the conclusions about the anisotropic, orbital dependent QPSW). As shown, those gaps exhibit a well-defined tendency: the gap maxima increase linearly with $T_{c}$, whereas the gap minima seem to decrease with $T_{c}$, leading to more isotropic order parameters in more disordered samples with lower $T_{c}$. This behavior is in excellent qualitative agreement with the systematic study of disorder in irradiated crystals by Sun et al. [17].

Figure 6(b) displays the gap functions deduced from the fits to all data (London penetration depth and specific heat), in comparison with the reference measurements of Sprau et al. [11]. The shaded area is the result from PCAR measurements. As shown, a very reasonable agreement is found between the three experimental techniques, as well as with the various data sets from the literature. Still, let us discuss the (small) discrepancies in the gap functions obtained from specific heat and from London penetration depth measurements [Fig. 6(b)]. London penetration depth was measured by TDO in Ref. [20] and by optical conductivity in Ref. [19], which are basically transport measurements. It is reasonable then to expect the signal to be highly sensitive to nematic twin boundaries, where the local gap functions were shown to exhibit reduced maxima and promote a nodeless behavior compared to the bulk $[39,40]$. Specific heat, on the other hand, is a bulk thermodynamic measurement, in which the relative contribution of twin boundaries to the signal is expected to be smaller. The discrepancies between specific heat and penetration depth measurements can then be naturally ascribed to twin boundaries. The results from PCAR measurements support this interpretation: a different number of twin boundaries in the vicinity of the contact is expected from one measurement to another, leading to a spread of SW and gap functions between the $C_{P}(T)$ and $\lambda(T)$ values.

In conclusion, this work strongly suggests that the normal state and superconducting state properties of $\mathrm{FeSe}$ are driven by the strong anisotropy of the quasiparticle spectral weight at the Fermi surface. As this anisotropy was suggested to result from orbital-dependent correlations in the Fe $3 d$ manifold, the same effects may be expected to be relevant in many other multiband superconductors with non-negligible electron correlations.

\section{ACKNOWLEDGMENTS}

The authors thank Prof. Davis for kindly providing us with the TB computer code. H.C. would like to thank J. Petrucci and S. Hudson for their fruitful inspiration. This research was performed using funding from the Laboratoire d'excellence LANEF (No. ANR-10-LABX-51-01), Institut Néel of the Centre National de la Recherche Scientifique, and Université Grenoble Alpes. This work was supported by the French National Research Agency through Grant No. ANR-12-JS040003-01 SUBRISSYME.
[1] G. R. Stewart, Rev. Mod. Phys. 83, 1589 (2011); P. Dai, ibid. 87, 855 (2015); Y. Bang and G. R. Stewart, J. Phys.: Condens. Matter 29, 12 (2017), and references therein.

[2] S. Mukherjee, A. Kreisel, P. J. Hirschfeld, and B. M. Andersen, Phys. Rev. Lett. 115, 026402 (2015); A. Fedorov, A. Yaresko, T. K. Kim, Y. Kushnirenko, E. Haubold, T. Wolf, M. Hoesch, A. Gruneis, B. Buchner, and S. V. Borisenko, Sci. Rep. 6, 36834 (2016).

[3] A. E. Bohmer, T. Arai, F. Hardy, T. Hattori, T. Iye, T. Wolf, H. V. Lohneisen, K. Ishida, and C. Meingast, Phys. Rev. Lett. 114, 027001 (2015).

[4] M. Abdel-Hafiez, Y. J. Pu, J. Brisbois, R. Peng, D. L. Feng, D. A. Chareev, A. V. Silhanek, C. Krellner, A. N. Vasiliev, and X.-J. Chen, Phys. Rev. B 93, 224508 (2016).

[5] M. Aichhorn, S. Biermann, T. Miyake, A. Georges, and M. Imada, Phys. Rev. B 82, 064504 (2010).

[6] Y. Yamakawa, S. Onari, and H. Kontani, Phys. Rev. X 6, 021032 (2016)

[7] M. D. Watson, S. Backes, A. A. Haghighirad, M. Hoesch, T. K. Kim, A. I. Coldea, and R. Valenti, Phys. Rev. B 95, 081106(R) (2017); M. D. Watson, A. A. Haghighirad, L. C. Rhodes, M. Hoesch, and T. K. Kim, New J. Phys. 19, 103021 (2017).
[8] L. Fanfarillo, J. Mansart, P. Toulemonde, H. Cercellier, P. Le Fèvre, F. Bertran, B. Valenzuela, L. Benfatto, and V. Brouet, Phys. Rev. B 94, 155138 (2016).

[9] A. Kreisel, B. M. Andersen, P. O. Sprau, A. Kostin, J. C. S. Davis, and P. J. Hirschfeld, Phys. Rev. B 95, 174504 (2017).

[10] A. Kreisel, B. M. Andersen, and P. J. Hirschfeld, Phys. Rev. B 98, 214518 (2018).

[11] P. O. Sprau, A. Kostin, A. Kreisel, A. E. Bohmer, V. Taufour, P. C. Canfield, S. Mukherjee, P. J. Hirschfeld, B. M. Andersen, and J. C. Seamus Davis, Science 357, 75 (2017).

[12] J.-Y. Lin, Y. S. Hsieh, D. A. Chareev, A. N. Vasiliev, Y. Parsons, and H. D. Yang, Phys. Rev. B 84, 220507(R) (2011); P. Bourgeois-Hope, S. Chi, D. A. Bonn, R. Liang, W. N. Hardy, T. Wolf, C. Meingast, N. Doiron-Leyraud, and L. Taillefer, Phys. Rev. Lett. 117, 097003 (2016).

[13] M. Abdel-Hafiez, J. Ge, A. N. Vasiliev, D. A. Chareev, J. Van de Vondel, V. V. Moshchalkov, and A. V. Silhanek, Phys. Rev. B 88, 174512 (2013); M. Abdel-Hafiez, Y.-Y. Zhang, Z.-Y. Cao, C.-G. Duan, G. Karapetrov, V. M. Pudalov, V. A. Vlasenko, A. V. Sadakov, D. A. Knyazev, T. A. Romanova, D. A. Chareev, O. S. Volkova, A. N. Vasiliev, and X.-J. Chen, ibid. 91, 165109 
(2015); M. D. Watson, T. Yamashita, S. Kasahara, W. Knafo, M. Nardone, J. Beard, F. Hardy, A. McCollam, A. Narayanan, S. F. Blake, T. Wolf, A. A. Haghighirad, C. Meingast, A. J. Schofield, H. V. Lohneysen, Y. Matsuda, A. I. Coldea, and T. Shibauchi, Phys. Rev. Lett. 115, 027006 (2015).

[14] L. Jiao, C.-L. Huang, S. Rossler, C. Koz, U. K. Rossler, U. Schwarz, and S. Wirth, Sci. Rep. 7, 44024 (2017).

[15] G.-Y. Chen, X. Zhu, H. Yang, and H.-H. Wen, Phys. Rev. B 96, 064524 (2017).

[16] Y. Sun, S. Kittaka, S. Nakamura, T. Sakakibara, K. Irie, T. Nomoto, K. Machida, J. Chen, and T. Tamegai, Phys. Rev. B 96, 220505(R) (2017).

[17] Y. Sun, A. Park, S. Pyon, T. Tamegai, and H. Kitamura, Phys. Rev. B 96, 140505(R) (2017).

[18] Y. Sun, S. Kittaka, S. Nakamura, T. Sakakibara, P. Zhang, S. Shin, K. Irie, T. Nomoto, K. Machida, J. Chen, and T. Tamegai, Phys. Rev. B 98, 064505 (2018).

[19] M. Li, N. R. Lee-Hone, S. Chi, R. Liang, W. N. Hardy, D. A. Bonn, E. Girt, and D. M. Broun, New J. Phys. 18, 082001 (2016).

[20] S. Teknowijoyo, K. Cho, M. A. Tanatar, J. Gonzales, A. E. Bohmer, O. Cavani, V. Mishra, P. J. Hirschfeld, S. L. Bud'ko, P. C. Canfield, and R. Prozorov, Phys. Rev. B 94, 064521 (2016).

[21] F. Hardy, M. He, L. Wang, T. Wolf, P. Schweiss, M. Merz, M. Barth, P. Adelmann, R. Eder, A.-A. Haghighirad, and C. Meingast, Phys. Rev. B 99, 035157 (2019).

[22] S. Karlsson, P. Strobel, A. Sulpice, C. Marcenat, M. Legendre, F. Gay, S. Pairis, O. Leynaud, and P. Toulemonde, Supercond. Sci. Technol. 28, 105009 (2015).

[23] A. Audouard, L. Drigo, F. Duc, P. Toulemonde, S. Karlsson, P. Strobel, and A. Sulpice, Europhys. Lett. 109, 27003 (2015).

[24] A. E. Bohmer, V. Taufour, W. E. Straszheim, T. Wolf, and P. C. Canfield, Phys. Rev. B 94, 024526 (2016).

[25] R. Prozorov and R. W. Giannetta, Supercond. Sci. Technol. 19, R41 (2006).

[26] Yu. G. Naidyuk and I. K. Yanson, Point-Contact Spectroscopy, Springer Series in Solid State Science (Springer, Berlin, 2005); A. G. M. Jansen, A. P. van Gelder, and P. Wyder, J. Phys. C: Solid. St. Phys. 13, 6073 (1980).

[27] D. Daghero and R. S. Gonnelli, Supercond. Sci. Technol. 23, 043001 (2010).

[28] D. Daghero, M. Tortello, G. A. Ummarino, and R. S. Gonnelli, Rep. Prog. Phys. 74, 124509 (2011).
[29] A. Kostin, P. O. Sprau, A. Kreisel, Y. X. Chong, A. E. Bohmer, P. C. Canfield, P. J. Hirschfeld, B. M. Andersen, and J. C. Séamus Davis, Nat. Mater. 17, 869 (2018).

[30] See Supplemental Material at http://link.aps.org/supplemental/ 10.1103/PhysRevB.100.104516 for extensive details of the calculations.

[31] M. D. Watson, T. K. Kim, A. A. Haghighirad, S. F. Blake, N. R. Davies, M. Hoesch, T. Wolf, and A. I. Coldea, Phys. Rev. B 92, 121108(R) (2015); C. H. P. Wen, H. C. Xu, C. Chen, Z. C. Huang, X. Lou, Y. J. Pu, Q. Song, B. P. Xie, M. Abdel-Hafiez, D. A. Chareev, A. N. Vasiliev, R. Peng, and D. L. Feng, Nat. Commun. 7, 10840 (2016).

[32] T. Hanaguri, S. Niitaka, K. Kuroki, and H. Takagi, Science 328, 474 (2010).

[33] S. Kasahara, T. Watashige, T. Hanaguri, Y. Kohsaka, T. Yamashita, Y. Shimoyama, Y. Mizukami, R. Endo, H. Ikeda, K. Aoyama, T. Terashima, S. Uji, T. Wolf, H. V. Lohneysen, T. Shibauchi, and Y. Matsuda, Proc. Natl. Acad. Sci. USA 111, 16309 (2014).

[34] Yu. G. Naidyuk, G. Fuchs, D. A. Chareev, and A. N. Vasiliev, Phys. Rev. B 93, 144515 (2016).

[35] G. E. Blonder, M. Tinkham, and T. M. Klapwijk, Phys. Rev. B 25, 4515 (1982).

[36] C.-L. Song, Y.-L. Wang, Y.-P. Jiang, Z. Li, L. Wang, K. He, X. Chen, J. E. Hoffman, X.-C. Ma, and Q.-K. Xue, Phys. Rev. Lett. 112, 057002 (2014).

[37] Q. Wang, Y. Shen, B. Pan, Y. Hao, M. Ma, F. Zhou, P. Steffens, K. Schmalzl, T. R. Forrest, M. Abdel-Hafiez, X. Chen, D. A. Chareev, A. N. Vasiliev, P. Bourges, Y. Sidis, H. Cao, and J. Zhao, Nat. Mater. 15, 159 (2016).

[38] L. C. Rhodes, M. D. Watson, A. A. Haghighirad, D. V. Evtushinsky, M. Eschrig, and T. K. Kim, Phys. Rev. B 98, 180503(R) (2018); D. Liu, C. Li, J. Huang, B. Lei, L. Wang, X. Wu, B. Shen, Q. Gao, Y. Zhang, X. Liu, Y. Hu, Y. Xu, A. Liang, J. Liu, P. Ai, L. Zhao, S. He, L. Yu, G. Liu, Y. Mao, X. Dong, X. Jia, F. Zhang, S. Zhang, F. Yang, Z. Wang, Q. Peng, Y. Shi, J. Hu, T. Xiang, X. Chen, Z. Xu, C. Chen, and X. J. Zhou, Phys. Rev. X 8, 031033 (2018).

[39] C.-L. Song, Y.-L. Wang, Y.-P. Jiang, L. Wang, K. He, X. Chen, J. E. Hoffman, X.-C. Ma, and Q.-K. Xue, Phys. Rev. Lett. 109, 137004 (2012).

[40] T. Watashige, Y. Tsutsumi, T. Hanaguri, Y. Kohsaka, S. Kasahara, A. Furusaki, M. Sigrist, C. Meingast, T. Wolf, H. V. Lohneysen, T. Shibauchi, and Y. Matsuda, Phys. Rev. X 5, 031022 (2015). 